\title{
Study of two-spin entanglement in singlet states
}

\author{
M. Q. Lone, A. Dey, S. Yarlagadda* \\ CMP Division, Saha Institute of Nuclear physics \\ 1/AF Salt Lake, Kolkata 700064, India
}

\begin{abstract}
We study the entanglement properties of two-spin subsystems in spin-singlet states. The average entanglement between two spins is maximized in a single valence-bond (VB) state. On the other hand, $E_{v}^{2}$ (the average entanglement between a subsystem of two spins and the rest of the system) can be maximized through a homogenized superposition of the VB states. The maximal $E_{v}^{2}$ rapidly increases with system size and saturates at its maximum allowed value. We adopt two ways of obtaining maximal $E_{v}^{2}$ states: (1) imposing homogeneity on singlet states; and (2) generating isotropy in a general homogeneous state. By using these two approaches, we construct explicitly four-spin and six-spin highly entangled states that are both isotropic and homogeneous. Our maximal $E_{v}^{2}$ states represent a new class of resonatingvalence-bond states which we show to be the ground states of the infiniterange Heisenberg model.
\end{abstract}

Keywords: D. Singlet states, D. RVB states, D. Entanglement, A. Frustrated magnets

\section{Introduction}

Valence-bond states were shown to be the ground states of spin systems earlier by Majumdar's group [1] and later by Shastry and Sutherland [2]. Any spin-singlet state (i.e., state with total spin eigenvalue $S_{T}=0$ ) can be expressed as a superposition of VB states [3, 4]. Spin-singlet states are important in understanding many problems in condensed matter physics as

\footnotetext{
${ }^{*}$ Corresponding author

Email address: y.sudhakar@saha.ac.in (S. Yarlagadda)
} 
well as in quantum information science. Resonating-valence-bond (RVB) singlet states have provided interesting insights for understanding strongly correlated phenomena such as spin liquid physics in frustrated magnets [5], physics of high $T_{c}$ cuprates [6, 7, 8], superconductivity in organic solids [9], insulator superconductor transition in boron-doped diamond [10], etc. Furthermore, RVB states have also been proposed as robust basis states for topological quantum computation [11]. As regards examples of real systems, valence-bond state (comprised of alternating dimer spin chain) has been experimentally realized in the spin-chain compound, copper nitrate $\left(\mathrm{Cu}\left(\mathrm{NO}_{3}\right)_{2} \times 2.5 \mathrm{H}_{2} \mathrm{O}\right)[12] ; \mathrm{RVB}$ state occurs naturally in $\mathrm{SrCu}_{2}\left(\mathrm{BO}_{3}\right)_{2}[13]$ which can be explained by the Shastry-Sutherland model [14]; valence bond solid has been observed in $\mathrm{Zn}_{\mathrm{x}} \mathrm{Cu}_{4-\mathrm{x}}(\mathrm{OD})_{6} \mathrm{Cl}_{2}$ [15].

Correlation functions of observables (such as density, magnetization, etc.) reflect the degree of entanglement in the pure state of a many-body system [16]. Quantum algorithms that would significantly accelerate a classical computation must rely on highly entangled states since slightly entangled states can be simulated efficiently on a classical computer [17]. Thus, characterization of multi-particle entanglement and production of maximal entanglement is vital for quantum computational studies and for mutual enrichment of quantum information science and many-body condensed matter physics.

Correlation/entanglement between two spins plays an important role in understanding phase transitions, length scale in the system, etc. Although two-spin correlation/entanglement has been investigated in certain RVB states [18, 4], to our knowledge, there has been no explicit construction of RVB states that would contain maximal entanglement of two-spin subsystems. The spins of a two-spin singlet, while being maximally entangled with each other, are completely unentangled with the remaining spins and thus show monogamy. Thus, if we wish to establish greater entanglement between the two-spin subsystem and the rest of the spin system, we are forced to diminish entanglement between the spins of the two-spin subsystem. The purpose of the present paper is to enhance our understanding of the distribution of twospin entanglement in singlet states. We analyze the following two extreme cases in a general singlet: (1) maximal average entanglement between two spins; and (2) maximal average entanglement between a two-spin subsystem and the remaining spins. The main results of this paper are as follows. First, we study two-spin entanglement in singlets. We show that the average entanglement between two spins is maximum (as expected) for a single VB state. In a singlet, we also demonstrate that $\mathrm{SU}(2)$ isotropy and homogene- 
ity (in spin-spin correlation function) maximize the bipartite entanglement $E_{v}^{2}$ while minimizing the average entanglement between two spins. Second, based on the principles of isotropy and homogeneity, we propose two approaches to construct entangled states that maximize $E_{v}^{2}$ and are a new class of RVB states. Last, we analyze these states in terms of ground states of the infinite-range Heisenberg model (IRHM).

\section{Entanglement for two spins calculated from reduced density ma- trix}

For a bipartite system $\mathrm{AB}$ in a pure state, von Neumann entropy $E_{v}$ measures the entanglement between the subsystems A and B. From the reduced density matrices $\rho_{A} \equiv \operatorname{Tr}_{B} \rho^{A B}$ and $\rho_{B} \equiv \operatorname{Tr}_{A} \rho^{A B}$, obtained from the pure state $\rho^{A B}$, we get the following:

$$
\begin{aligned}
E_{v}\left(\rho_{A}\right) & =-\operatorname{Tr}\left(\rho_{A} \log _{2} \rho_{A}\right) \\
& =-\operatorname{Tr}\left(\rho_{B} \log _{2} \rho_{B}\right)=E_{v}\left(\rho_{B}\right) .
\end{aligned}
$$

Using $S^{i}=\frac{1}{2} \sigma^{i}$ and the basis $|\downarrow\rangle$ and $|\uparrow\rangle$, the single spin (reduced) density matrix can be written as [20]

$$
\rho_{i}=\left[\begin{array}{cc}
\frac{1}{2}-\left\langle S_{i}^{z}\right\rangle & \left\langle S_{i}^{+}\right\rangle \\
\left\langle S_{i}^{-}\right\rangle & \frac{1}{2}+\left\langle S_{i}^{z}\right\rangle
\end{array}\right],
$$

where $S^{ \pm} \equiv S^{x} \pm i S^{y}$. For SU(2) singlet states, since the z-component of the total spin operator $\left(S_{\text {Total }}^{z}\right)$ has eigenvalue $S_{T}^{z}=0,\left\langle S_{i}^{+}\right\rangle=0$. Then, from the isotropy of the singlet states, it follows that $\left\langle S_{i}^{z}\right\rangle=0$. Consequently, the single-spin density matrix becomes maximally mixed and the entanglement $E_{v}\left(\rho_{i}\right)$ becomes maximized.

We will now show that the average entanglement between a pair of spins is maximum for a VB state. To this end, we obtain an expression for the tangle which is a measure of entanglement. For a pure state, the tangle between the spin at $i$ and the rest of the spins is given by $\tau\left(\rho_{i}\right)=2\left[1-\operatorname{Tr}\left(\rho_{i}^{2}\right)\right]$ [21]; from Eq. (2), it follows that $\tau\left(\rho_{i}\right)=1$ for a spin singlet. Next, we will deal with the tangle between spins at $i$ and $j$ for the mixed state $\rho_{i j}$ (i.e., the two-spin reduced density matrix). The tangle $\tau\left(\rho_{i j}\right)$ is given by the square of $\max \left\{\lambda_{1}-\right.$ $\left.\lambda_{2}-\lambda_{3}-\lambda_{4}, 0\right\}$ where $\lambda_{1}, \lambda_{2}, \lambda_{3}$, and $\lambda_{4}$ are the square roots of the eigenvalues (in decreasing order) of the operator $\rho_{i j}\left(\sigma_{y} \otimes \sigma_{y}\right) \rho_{i j}^{\star}\left(\sigma_{y} \otimes \sigma_{y}\right)$ where the asterisk corresponds to complex conjugation in the basis $\{|00\rangle,|01\rangle,|10\rangle,|11\rangle\}$. 
Now, the distribution of the bipartite entanglement (as measured by the tangle) amongst $\mathrm{N}$ spins satisfies the following inequality [21]:

$$
\sum_{j \neq i} \tau\left(\rho_{i j}\right) \leq \tau\left(\rho_{i}\right)
$$

For a VB state, it is straight forward to show that the tangle $\tau\left(\rho_{i j}\right)=1$ when the spins at $i$ and $j$ form a singlet and that $\tau\left(\rho_{i j}\right)=0$ otherwise. Thus, we see that a VB state satisfies $\sum_{j \neq i} \tau\left(\rho_{i j}\right)=\tau\left(\rho_{i}\right)$ for all $i$. Hence, in a VB state, the average entanglement between two spins $[1 / N(N-1)] \sum_{i, j \neq i} \tau\left(\rho_{i j}\right)$ attains the maximum allowed valued of 1 .

We will now proceed to obtain the condition for maximal average entanglement between a two-spin subsystem and the rest of the spins in a singlet. On realizing that $\left\langle S_{i}^{z}\right\rangle=0$, we obtain the following expression for the twospin reduced density matrix [20]:

$$
\rho_{i j}=\left[\begin{array}{cccc}
\frac{1}{4}+\left\langle S_{i}^{z} S_{j}^{z}\right\rangle & 0 & 0 & 0 \\
0 & \frac{1}{4}-\left\langle S_{i}^{z} S_{j}^{z}\right\rangle & \left\langle S_{i}^{+} S_{j}^{-}\right\rangle & 0 \\
0 & \left\langle S_{i}^{-} S_{j}^{+}\right\rangle & \frac{1}{4}-\left\langle S_{i}^{z} S_{j}^{z}\right\rangle & 0 \\
0 & 0 & 0 & \frac{1}{4}+\left\langle S_{i}^{z} S_{j}^{z}\right\rangle
\end{array}\right] .
$$

Here, isotropy implies that $0.5\left\langle S_{i}^{-} S_{j}^{+}\right\rangle=0.5\left\langle S_{i}^{+} S_{j}^{-}\right\rangle=\left\langle S_{i}^{x} S_{j}^{x}\right\rangle=\left\langle S_{i}^{y} S_{j}^{y}\right\rangle=$ $\left\langle S_{i}^{z} S_{j}^{z}\right\rangle$. Thus, the von Neumann entropy $E_{v}\left(\rho_{i j}\right)$ can be expressed as

$$
\begin{aligned}
E_{v}\left(\rho_{i j}\right)=2-\frac{1}{4}[ & 3\left(1+4\left\langle S_{i}^{z} S_{j}^{z}\right\rangle\right) \log _{2}\left(1+4\left\langle S_{i}^{z} S_{j}^{z}\right\rangle\right) \\
+ & \left.\left(1-12\left\langle S_{i}^{z} S_{j}^{z}\right\rangle\right) \log _{2}\left(1-12\left\langle S_{i}^{z} S_{j}^{z}\right\rangle\right)\right] .
\end{aligned}
$$

For our states, since $S_{\text {Total }}^{z}|\Psi\rangle=0$, we observe that

$$
\sum_{j \neq i}\left\langle S_{i}^{z} S_{j}^{z}\right\rangle=-\left\langle S_{i}^{z 2}\right\rangle=-\frac{1}{4} .
$$

We will now maximize the total entanglement entropy $\sum_{i, j \neq i} E_{v}\left(\rho_{i j}\right)$ subject to the constraint in Eq. ([6). To this end, we will employ the method of Lagrange multipliers and define the Lagrange function $\Lambda$ as follows:

$$
\Lambda=\sum_{i, j \neq i} E_{v}\left(\rho_{i j}\right)-\sum_{i} \lambda_{i}\left(\sum_{j \neq i}\left\langle S_{i}^{z} S_{j}^{z}\right\rangle+\frac{1}{4}\right) .
$$


Then, setting $\frac{\partial \Lambda}{\partial\left\langle S_{l}^{z} S_{m}^{z}\right\rangle}=0$ yields

$$
\lambda_{l}=3 \log _{2}\left[\frac{\left(1-12\left\langle S_{l}^{z} S_{m}^{z}\right\rangle\right)}{\left(1+4\left\langle S_{l}^{z} S_{m}^{z}\right\rangle\right)}\right],
$$

which implies that the optimal $\left\langle S_{l}^{z} S_{m}^{z}\right\rangle$ is independent of $m$ for all $m \neq l$. Consequently, it follows from Eq. (6) that $\sum_{i, j \neq i} E_{v}\left(\rho_{i j}\right)$ is maximized when $\left\langle S_{i}^{z} S_{j}^{z}\right\rangle=-\frac{1}{4(N-1)}$, i.e., when the isotropic state has a homogeneous longitudinal spin-spin correlation function. The average entanglement entropy between the subsystem of two spins and the remaining $N-2$ spins, expressed as $E_{v}^{2} \equiv[1 / N(N-1)] \sum_{i, j \neq i} E_{v}\left(\rho_{i j}\right)$, has a maximum value given by

$$
\begin{aligned}
E_{v, \text { max }}^{2}(N)= & -3\left(\frac{1}{4}-\frac{1}{4(N-1)}\right) \log _{2}\left(\frac{1}{4}-\frac{1}{4(N-1)}\right) \\
& -\left(\frac{1}{4}+\frac{3}{4(N-1)}\right) \log _{2}\left(\frac{1}{4}+\frac{3}{4(N-1)}\right) .
\end{aligned}
$$

It is interesting to note that for $N \rightarrow \infty$, the above expression yields $E_{v}^{2} \rightarrow 2$. In fact, $E_{v}^{2}$ approaches the maximum possible value 2 quite rapidly as can be seen from Fig. 1. Next, for $N=4$, we observe that our expression for $E_{v, \max }^{2}$ in Eq. (9) yields the same entanglement entropy value $1+0.5 \log _{2} 3$ as that obtained for the four-spin maximally entangled state studied in Refs. [22, 23].

We also note that homogeneity in the two-spin correlation function $\left\langle S_{i}^{z} S_{j}^{z}\right\rangle$, under the constraint of Eq. ([6), maximizes the average entanglement (between a two-spin subsystem and the rest of the system) as measured by i-concurrence $I_{c}$ given below [24]:

$$
\mathrm{I}_{\mathrm{c}}=\frac{2}{N(N-1)} \sum_{i, j>i} \sqrt{2\left[1-\operatorname{Tr}\left(\rho_{i j}^{2}\right)\right]} .
$$

As shown in Fig. 1, $\mathrm{I}_{c, \max }$ also monotonically increases with system size.

Lastly, we mention that the tangle $\tau\left(\rho_{i j}\right)$ (between spins at $i$ and $j$ ) for a singlet [on using the definition and Eq. (4)] is given by square of $\max \left\{4\left|\left\langle S_{i}^{z} S_{j}^{z}\right\rangle\right|-2\left\langle S_{i}^{z} S_{j}^{z}\right\rangle-1 / 2,0\right\}$. For the singlets that have $E_{v}^{2}$ maximized (which occurs when $\left.\left\langle S_{i}^{z} S_{j}^{z}\right\rangle=-\frac{1}{4(N-1)}\right)$, the tangle $\tau\left(\rho_{i j}\right)=0$ when $N \geq 4$. Hence, the average entanglement between spins at sites $i$ and $j$ is given by $[1 / N(N-1)] \sum_{i, j \neq i} \tau\left(\rho_{i j}\right)=0$. 


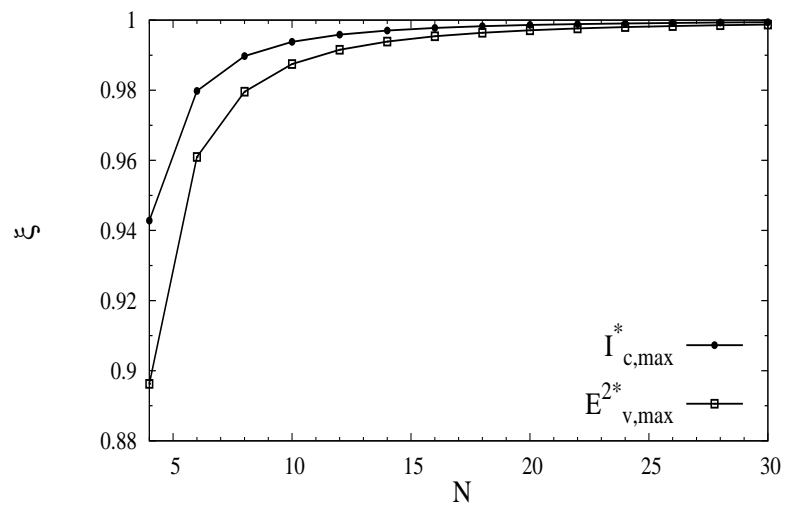

Figure 1: Normalized entanglement $\xi$, for the two-spin reduced density matrix, measured by (a) von Neumann entropy $\left[\mathrm{E}_{\mathrm{v}, \max }^{2 \star} \equiv \mathrm{E}_{\mathrm{v}, \max }^{2}(\mathrm{~N}) / \mathrm{E}_{\mathrm{v}, \max }^{2}(\infty)\right]$ and (b) i-concurrence $\left[\mathrm{I}_{\mathrm{c}, \max }^{\star} \equiv \mathrm{I}_{\mathrm{c}, \max }(\mathrm{N}) / \mathrm{I}_{\mathrm{c}, \max }(\infty)\right]$, for $\mathrm{N}$-spin VB systems.

Our maximal $E_{v}^{2}$ states can be regarded as a new class of RVB states made of homogenized superposition of valence-bond states. In the related work of Ref. [4], an interesting analysis of entanglement between two sites was carried out in two different RVB systems, i.e., the RVB gas involving equal-amplitude superposition of all bipartite VB coverings and the RVB liquid involving equal-amplitude superposition of all nearest-neighbor-singlet VB coverings of a lattice. The RVB gas, for finite-size systems, revealed a non-zero tangle between two sites. On the other hand, the RVB liquid, for small systems, i.e., a $4 \times 4$ lattice, manifests zero (non-zero) tangle between two sites for open (periodic) boundary conditions [4].

\section{Maximal $E_{v}^{2}$ states}

In this section, we offer two different approaches for constructing maximal $E_{v}^{2}$ states. The first approach involves producing homogeneity in isotropic states while the second deals with generating isotropy in homogeneous states. As examples of our prescribed procedure, we construct highly entangled states for four spins and six spins.

\subsection{Generating homogeneity in isotropic states}

In this section, we will proceed to construct entangled states for N-spins that maximize $E_{v}^{2}$. We first note that there are $(N-1)$ !! states with $S_{T}=0$ 
and each of these is a product of $N / 2$ two-spin singlet states of the form $\mid \uparrow \downarrow$ \rangle$-|\downarrow \uparrow\rangle$. Of these $(N-1)$ !! product combinations with $S_{T}=0$, only ${ }^{N} C_{\frac{N}{2}}-{ }^{N} C_{\frac{N}{2}-1}=N ! /[(N / 2) !(N / 2+1) !]$ products are linearly independent [1]. A particular set of linearly-independent $S_{T}=0$ states are the Rumer states that are made up of non-crossing singlets [1, 2].

Next, we show how to construct highly entangled states by starting with isotropic states and making them homogeneous. Since we are dealing with spin-singlets, we consider $\frac{N}{2} \uparrow$ spins and $\frac{N}{2} \downarrow$ spins in our basis states $\left|\psi_{k}^{z}\right\rangle$ ( $\equiv\left|\sigma_{1}^{z} \sigma_{2}^{z} \ldots \sigma_{N}^{z}\right\rangle$ with $\sigma_{i}^{z}=\uparrow$ or $\downarrow$ ). Using superposition of these basis states, with all the basis states being equally probable, we construct the general homogeneous states

$$
\left|\Psi_{N}\right\rangle_{h o m}=\sum_{k=1}^{{ }^{N} C_{N / 2}} e^{i \delta_{k}}\left|\psi_{k}^{z}\right\rangle,
$$

which we will now prove to yield $\left\langle S_{i}^{z} S_{j}^{z}\right\rangle=-\frac{1}{4(N-1)}$. Throughout this paper, for convenience, we ignore the normalization constants in our spin states. Let the spin at site $i$ be either $\uparrow$ or $\downarrow$. Then, the probability that the spin at site $j \neq i$ is in the same state is $\frac{[(N / 2)-1]}{[(N / 2)-1]+N / 2}$, whereas the probability that it is in the opposite state is $\frac{[N / 2]}{[(N / 2)-1]+N / 2}$. Therefore, we can write

$$
\begin{aligned}
\left\langle S_{i}^{z} S_{j}^{z}\right\rangle & =\frac{1}{4}\left[\frac{\frac{N}{2}-1}{\left(\frac{N}{2}-1\right)+\frac{N}{2}}\right]-\frac{1}{4}\left[\frac{\frac{N}{2}}{\left(\frac{N}{2}-1\right)+\frac{N}{2}}\right] \\
& =-\frac{1}{4(N-1)} .
\end{aligned}
$$

Thus, when all the basis states are equally probable, we get $\left\langle S_{i}^{z} S_{j}^{z}\right\rangle=$ $-\frac{1}{4(N-1)}$. Now, we are in a position to form (from isotropic $S_{T}=0$ states) homogenized states that are highly entangled.

\subsubsection{Maximal $E_{v}^{2}$ states for four spins}

For $N=4$ spins, we have two linearly-independent, non-crossing Rumer diagrams which are shown in Fig. 2. The states corresponding to these diagrams can be expressed as $\left|\Phi_{12}^{S_{12}=0}\right\rangle \otimes\left|\Phi_{34}^{S_{34}=0}\right\rangle$ and $\left|\Phi_{14}^{S_{14}=0}\right\rangle \otimes\left|\Phi_{23}^{S_{23}=0}\right\rangle$ where $\left|\Phi_{i j}^{S_{i j}=0}\right\rangle \equiv\left[|\uparrow\rangle_{i}|\downarrow\rangle_{j}-|\downarrow\rangle_{i}|\uparrow\rangle_{j}\right]$ is a two-spin singlet state with $S_{i j}$ being the total spin of $S_{i}$ and $S_{j}$. It is worth noting that

$$
\begin{aligned}
\left|\Phi_{13}^{S_{13}=0}\right\rangle \otimes\left|\Phi_{24}^{S_{24}=0}\right\rangle= & \left|\Phi_{12}^{S_{12}=0}\right\rangle \otimes\left|\Phi_{34}^{S_{34}=0}\right\rangle \\
& +\left|\Phi_{14}^{S_{14}=0}\right\rangle \otimes\left|\Phi_{23}^{S_{23}=0}\right\rangle,
\end{aligned}
$$




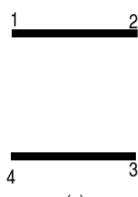

(a)

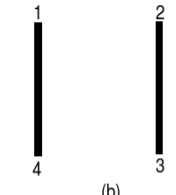

(b)

Figure 2: Linearly-independent, non-crossing Rumer diagrams for a four-spin system .

\begin{tabular}{|l|l|}
\hline Basis states $\left|\psi_{k}^{z}\right\rangle$ & Coefficients $e^{i \delta_{k}}$ in Eq. (11). \\
\hline$|\uparrow \downarrow \uparrow \downarrow\rangle$ & $r_{1} e^{i \theta_{1}}-r_{2} e^{i \theta_{2}}$ \\
\hline$|\downarrow \uparrow \downarrow \uparrow\rangle$ & $r_{1} e^{i \theta_{1}}-r_{2} e^{i \theta_{2}}$ \\
\hline$|\uparrow \downarrow \downarrow \uparrow\rangle$ & $-r_{1} e^{i \theta_{1}}$ \\
\hline$|\downarrow \uparrow \uparrow \downarrow\rangle$ & $-r_{1} e^{i \theta_{1}}$ \\
\hline$|\uparrow \uparrow \downarrow \downarrow\rangle$ & $r_{2} e^{i \theta_{2}}$ \\
\hline$|\downarrow \downarrow \uparrow \uparrow\rangle$ & $r_{2} e^{i \theta_{2}}$ \\
\hline
\end{tabular}

Table 1: Basis states $\left|\psi_{k}^{z}\right\rangle$ of Eq. (11) for a four-spin system and the corresponding coefficients $e^{i \delta_{k}}$ obtained by setting $\left|\Psi^{4}\right\rangle$ [in Eq. (14)] equal to $\left|\Psi_{N}\right\rangle_{h o m}$ [in Eq. (11)] with $N=4$.

which means that the crossing singlet $\left|\Phi_{13}^{S_{13}=0}\right\rangle \otimes\left|\Phi_{24}^{S_{24}=0}\right\rangle$ depends linearly on the two non-crossing singlets $\left|\Phi_{12}^{S_{12}=0}\right\rangle \otimes\left|\Phi_{34}^{S_{34}=0}\right\rangle$ and $\left|\Phi_{14}^{S_{14}=0}\right\rangle \otimes\left|\Phi_{23}^{S_{23}=0}\right\rangle$. Using the above relation, one can establish that there are only ${ }^{N} C_{\frac{N}{2}}-{ }^{N} C_{\frac{N}{2}-1}$ linearly-independent $S_{T}=0$ states.

Next, we take the following linear superposition to obtain the desired entangled state:

$$
\begin{aligned}
\left|\Psi^{4}\right\rangle & =r_{1} e^{i \theta_{1}}\left[\left|\Phi_{12}^{S_{12}=0}\right\rangle \otimes\left|\Phi_{34}^{S_{34}=0}\right\rangle\right] \\
& +r_{2} e^{i \theta_{2}}\left[\left|\Phi_{14}^{S_{14}=0}\right\rangle \otimes\left|\Phi_{23}^{S_{23}=0}\right\rangle\right],
\end{aligned}
$$

where $r_{i} e^{i \theta_{i}}$ represents a general coefficient. On setting $\left|\Psi^{4}\right\rangle=\left|\Psi_{N}\right\rangle_{\text {hom }}$ with $N=4$, as shown in Table 1, we get the various coefficients for each of the basis states $\left|\psi_{k}^{z}\right\rangle$ occurring in Eq. (11).

The expression for $\left|\Psi^{4}\right\rangle$ [in Eq. (14)] assumes the homogenized superposed form shown in Eq. (11) for two linearly-independent sets of solu- 


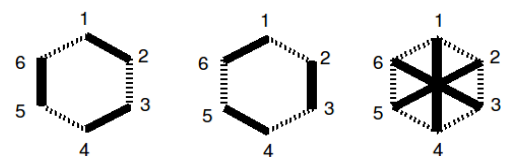

(a)

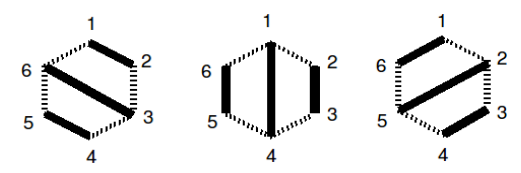

(b)

Figure 3: Homogenized linear combination of the five non-crossing $S_{T}=0$ singlet states (or non-crossing Rumer diagrams) in (a) and (b) give maximal $E_{v}^{2}$ entanglement for six spins. The state $\left|\Psi_{c}^{6}\right\rangle$ in Eq. (S8) is a linear combination of the three diagrams in (a). On the other hand, $\left|\Psi_{a}^{6}\right\rangle$ in Eq. (S5) and $\left|\Psi_{b}^{6}\right\rangle$ in Eq. (S6) represent different linear combinations of all the three diagrams in (b).

tions of the coefficients in Table 1. The two sets of solutions are $\left\{r_{1} e^{i \theta_{1}}=\right.$ $\left.-e^{i 2 \pi / 3}, r_{2} e^{i \theta_{2}}=e^{i 4 \pi / 3}\right\}$ and its complex conjugate. The states corresponding to these solutions are

$$
\begin{aligned}
&\left|\Psi^{4}\right\rangle= e^{i 2 \pi / 3}\left(\left|\Phi_{12}^{S_{12}=0}\right\rangle \otimes\left|\Phi_{34}^{S_{34}=0}\right\rangle\right) \\
&+ e^{i 4 \pi / 3}\left(\left|\Phi_{14}^{S_{14}=0}\right\rangle \otimes\left|\Phi_{23}^{S_{23}=0}\right\rangle\right) \\
&= {\left[(|\uparrow \downarrow \uparrow \downarrow\rangle+|\downarrow \uparrow \downarrow \uparrow\rangle)+\omega_{3}(|\uparrow \downarrow \downarrow \uparrow\rangle+|\downarrow \uparrow \uparrow \downarrow\rangle)\right.} \\
&\left.\quad+\omega_{3}^{2}(|\uparrow \uparrow \downarrow \downarrow\rangle+|\downarrow \downarrow \uparrow \uparrow\rangle)\right],
\end{aligned}
$$

and its complex conjugate. In the above equation, $\omega_{3}=e^{i 2 \pi / 3}$, i.e., a cube root of unity. The above state is the maximally entangled state for four spins studied in Refs. [22, 23].

\subsubsection{Maximal $E_{v}^{2}$ states for six spins}

The linearly-independent states in this case are five in number (i.e., the five non-crossing Rumer diagrams of Fig. 3). Thus, similar to the four-spin case, we begin with the following general linear superposition:

$$
\begin{aligned}
\left|\Psi^{6}\right\rangle & =r_{1} e^{i \theta_{1}}\left[\left|\Phi_{12}^{S_{12}=0}\right\rangle \otimes\left|\Phi_{34}^{S_{34}=0}\right\rangle \otimes\left|\Phi_{56}^{S_{56}=0}\right\rangle\right] \\
& +r_{2} e^{i \theta_{2}}\left[\left|\Phi_{61}^{S_{61}=0}\right\rangle \otimes\left|\Phi_{23}^{S_{23}=0}\right\rangle \otimes\left|\Phi_{45}^{S_{45}=0}\right\rangle\right]
\end{aligned}
$$




$$
\begin{aligned}
& +\quad r_{3} e^{i \theta_{3}}\left[\left|\Phi_{12}^{S_{12}=0}\right\rangle \otimes\left|\Phi_{36}^{S_{36}=0}\right\rangle \otimes\left|\Phi_{45}^{S_{45}=0}\right\rangle\right] \\
& +\quad r_{4} e^{i \theta_{4}}\left[\left|\Phi_{23}^{S_{23}=0}\right\rangle \otimes\left|\Phi_{14}^{S_{14}=0}\right\rangle \otimes\left|\Phi_{56}^{S_{56}=0}\right\rangle\right] \\
& +\quad r_{5} e^{i \theta_{5}}\left[\left|\Phi_{16}^{S_{16}=0}\right\rangle \otimes\left|\Phi_{25}^{S_{25}=0}\right\rangle \otimes\left|\Phi_{34}^{S_{34}=0}\right\rangle\right]
\end{aligned}
$$

and find the solutions that homogenize $\left|\Psi^{6}\right\rangle$, i.e., make $\left|\Psi^{6}\right\rangle$ assume the form of $\left|\Psi_{6}\right\rangle_{\text {hom }}$ in Eq. (11). Since there are only five linearly-independent Rumer states, we observe that we can construct at most five linearly-independent superpositions of the form of $\left|\Psi^{6}\right\rangle$ in Eq. (S4). A set of five linearly-independent solutions are as follows (with details given in Ref. [4]):

$$
\begin{aligned}
\left|\Psi_{a}^{6}\right\rangle= & \omega_{4}\left(\left|\Phi_{12}^{S_{12}=0}\right\rangle \otimes\left|\Phi_{36}^{S_{36}=0}\right\rangle \otimes\left|\Phi_{45}^{S_{45}=0}\right\rangle\right) \\
& +\omega_{4}^{2}\left(\left|\Phi_{23}^{S_{23}=0}\right\rangle \otimes\left|\Phi_{14}^{S_{14}=0}\right\rangle \otimes\left|\Phi_{56}^{S_{56}=0}\right\rangle\right) \\
& +\omega_{4}^{3}\left(\left|\Phi_{16}^{S_{16}=0}\right\rangle \otimes\left|\Phi_{25}^{S_{25}=0}\right\rangle \otimes\left|\Phi_{34}^{S_{34}=0}\right\rangle\right),
\end{aligned}
$$

and its complex conjugate $\left|\Psi_{a}^{6 *}\right\rangle$,

$$
\begin{aligned}
\left|\Psi_{b}^{6}\right\rangle= & -\left(\left|\Phi_{12}^{S_{12}=0}\right\rangle \otimes\left|\Phi_{36}^{S_{36}=0}\right\rangle \otimes\left|\Phi_{45}^{S_{45}=0}\right\rangle\right) \\
& +\left(\left|\Phi_{23}^{S_{23}=0}\right\rangle \otimes\left|\Phi_{14}^{S_{14}=0}\right\rangle \otimes\left|\Phi_{56}^{S_{56}=0}\right\rangle\right) \\
& -\left(\left|\Phi_{16}^{S_{16}=0}\right\rangle \otimes\left|\Phi_{25}^{S_{25}=0}\right\rangle \otimes\left|\Phi_{34}^{S_{34}=0}\right\rangle\right), \\
\left|\Psi_{c}^{6}\right\rangle= & \omega_{4}\left(\left|\Phi_{12}^{S_{12}=0}\right\rangle \otimes\left|\Phi_{34}^{S_{34}=0}\right\rangle \otimes\left|\Phi_{65}^{S_{65}=0}\right\rangle\right) \\
& +\omega_{4}^{2}\left(\left|\Phi_{14}^{S_{14}=0}\right\rangle \otimes\left|\Phi_{25}^{S_{25}=0}\right\rangle \otimes\left|\Phi_{36}^{S_{36}=0}\right\rangle\right) \\
& +\omega_{4}^{3}\left(\left|\Phi_{61}^{S_{61}=0}\right\rangle \otimes\left|\Phi_{23}^{S_{23}=0}\right\rangle \otimes\left|\Phi_{45}^{S_{45}=0}\right\rangle\right),
\end{aligned}
$$

and its complex conjugate $\left|\Psi_{c}^{6 *}\right\rangle$. The state in Eq. (S8) represents the superposition of the three diagrams in Fig. 3(a) with the second term in the sum representing crossing singlets.

\subsection{Producing isotropy in homogeneous states}

Next, we produce isotropy in a given homogeneous state. Such states should also yield the entanglement value $E_{v \max }^{2}$. The condition of isotropy in a homogeneous state can be expressed as $\sum_{i} S_{i}^{\alpha}\left|\Psi_{N}\right\rangle_{\text {hom }}=0$ with $\alpha=x, y$, or $z$; this implies that $\sum_{i} S_{i}^{+}\left|\Psi_{N}\right\rangle_{\text {hom }}=0$. Now, reflection about the z-axis transforms $\Phi_{i j}^{S_{i j}=0}$ to $-\Phi_{i j}^{S_{i j}=0}$. Then, in a $S_{T}=0$ state comprising of $\frac{N}{2}$ singlets, reflection leads to the coefficient $(-1)^{\frac{N}{2}}$ for the parent state. Hence, when isotropy is imposed on the homogeneous state of Eq. (11) and all 
the spins are flipped, the parent state acquires the coefficient $(-1)^{\frac{N}{2}}$. In fact, in Eq. (11), the coefficients of the basis state $\left|\sigma_{1}^{z} \sigma_{2}^{z} \ldots \sigma_{N}^{z}\right\rangle$ and its spin flipped version differ only by a factor $(-1)^{\frac{N}{2}}$. Thus, the number of unknown coefficients $e^{i \delta_{k}}$ in Eq. (11) is reduced to $0.5\left({ }^{N} C_{\frac{N}{2}}\right)$. This also implies that the condition $\sum_{i} S_{i}^{-}\left|\Psi_{N}\right\rangle_{\text {hom }}=0$ yields the same equations as the condition $\sum_{i} S_{i}^{+}\left|\Psi_{N}\right\rangle_{\text {hom }}=0$ does. Therefore, we can write the four-spin homogeneous state as

$$
\begin{aligned}
\left|\Psi_{4}\right\rangle_{h o m} & =e^{i \phi_{1}}[|\uparrow \downarrow \uparrow \downarrow\rangle+|\downarrow \uparrow \downarrow \uparrow\rangle] \\
& +\quad e^{i \phi_{2}}[|\uparrow \downarrow \downarrow \uparrow\rangle+|\downarrow \uparrow \uparrow \downarrow\rangle] \\
& +\quad e^{i \phi_{3}}[|\uparrow \uparrow \downarrow \downarrow\rangle+|\downarrow \downarrow \uparrow \uparrow\rangle] .
\end{aligned}
$$

Furthermore, in the above four-spin, homogeneous state, the condition of isotropy yields

$$
\begin{aligned}
\sum_{i=1}^{4} S_{i}^{+}\left|\Psi_{4}\right\rangle_{h o m} & =\left(e^{i \phi_{1}}+e^{i \phi_{2}}+e^{i \phi_{3}}\right)[|\uparrow \uparrow \uparrow \downarrow\rangle+|\uparrow \downarrow \uparrow \uparrow\rangle \\
& |\uparrow \uparrow \downarrow \uparrow\rangle+|\downarrow \uparrow \uparrow \uparrow\rangle] \\
& =0 .
\end{aligned}
$$

This leads to the expression $e^{i \phi_{1}}+e^{i \phi_{2}}+e^{i \phi_{3}}=0$, i.e., zero-valued coefficients for all the four basis states. There are only two independent solutions to this equation: $\left\{e^{i \phi_{1}}=1, e^{i \phi_{2}}=\omega_{3}, e^{i \phi_{3}}=\omega_{3}^{2}\right\}$ and its complex conjugate. The states obtained from these solutions are the same as those obtained earlier by imposing homogeneity on isotropic states, i.e., $\left|\Psi^{4}\right\rangle$ in Eq. (15) and its complex conjugate.

Next, we consider the case of a six-spin, homogeneous state and impose isotropy. We then generate the same entangled states $\left|\Psi_{a}^{6}\right\rangle,\left|\Psi_{a}^{6 *}\right\rangle,\left|\Psi_{b}^{6}\right\rangle,\left|\Psi_{c}^{6}\right\rangle$, and $\left|\Psi_{c}^{6 *}\right\rangle$ obtained in the previous section (see Ref. [4] for details). Lastly, for any of the states $\left|\Psi_{a}^{6}\right\rangle,\left|\Psi_{a}^{6 *}\right\rangle,\left|\Psi_{b}^{6}\right\rangle,\left|\Psi_{c}^{6}\right\rangle$, or $\left|\Psi_{c}^{6 *}\right\rangle$, we observe that the von Neumann entropy $E_{v}^{2}$ has the value 1.921928 which is the same as that given by $E_{v, \max }^{2}$ in Eq. (91).

\subsection{Discussion of the general case}

We will now discuss generating $E_{v, \max }^{2} \mathrm{RVB}$ states for the general case of $\mathrm{N}$-spin system. The number of coefficients $r_{i} e^{i \theta_{i}}$, needed to generate a superposed state $\left|\Psi^{N}\right\rangle$ using all the non-crossing Rumer states, is the same as 
the total number of linearly-independent Rumer states, i.e., ${ }^{N} C_{\frac{N}{2}}-{ }^{N} C_{\frac{N}{2}-1}$. The number of unknown coefficients $e^{i \delta_{k}}$ (for the basis states $\left|\psi_{k}^{z}\right\rangle$ ) in Eq. (11) is $0.5\left({ }^{N} C_{\frac{N}{2}}\right)$. On setting $\left|\Psi^{N}\right\rangle=\left|\Psi_{N}\right\rangle_{h o m}$ and equating the coefficients of the various basis states $\left|\psi_{k}^{z}\right\rangle$, we get $0.5\left({ }^{N} C_{\frac{N}{2}}\right)$ equations; from these equations, on eliminating $r_{i} e^{i \theta_{i}}$ in terms of the various $e^{i \delta_{k}}$, we get $-0.5\left({ }^{N} C_{\frac{N}{2}}\right)+{ }^{N} C_{\frac{N}{2}-1}$ number of linearly-independent equations in terms of $0.5\left({ }^{N} C_{\frac{N}{2}}\right)$ number of unknown coefficients $e^{i \delta_{k}}$. Thus, we expect the number of independent $E_{v, \max }^{2}$ RVB states to be equal to the number of unknown $e^{i \delta_{k}}$ minus the number of independent equations, i.e., ${ }^{N} C_{\frac{N}{2}}-{ }^{N} C_{\frac{N}{2}-1}$ which is the number of linearly-independent Rumer states. For instance, for $N=8$, we get 21 linearly-independent equations in terms of 35 different $e^{i \delta_{k}}$; hence, the number of independent $E_{v, \max }^{2}$ RVB states is $14(=35-21)$ which is the total number of non-crossing Rumer states. We also would like to point out that explicit construction of $E_{v, \max }^{2}$ RVB states becomes more and more cumbersome as the spin-system size $\mathrm{N}$ increases.

\section{Generating highly entangled ground states using IRHM}

In this section, we will demonstrate that the $E_{v, \text { max }}^{2}$ entangled states obtained earlier, from the homogenization of isotropic states or from imposing isotropy on homogeneous states, are the ground states of a spin Hamiltonian. To this end, we begin with the IRHM Hamiltonian

$$
H_{\mathrm{IRHM}}=J \sum_{i, j>i} \vec{S}_{i} \cdot \vec{S}_{j}=\frac{J}{2}\left[\left(\sum_{i} \vec{S}_{i}\right)^{2}-\left(\sum_{i} \vec{S}_{i}^{2}\right)\right],
$$

and show that certain superpositions of the ground states of IRHM will produce the same amount of entanglement as given by Eq. (91). We observe that $\left[S_{\text {Total }}^{z}, H_{\text {IRHM }}\right]=0$ and that $\left[S_{\text {Total }}^{2}, H_{\text {IRHM }}\right]=0$. In Eq. (22), we take $J=J^{\star} /(N-1)$ (where $J^{\star}$ is a finite quantity) so that the energy per site remains finite as $N \rightarrow \infty$. The eigenstates of $H_{\text {IRHM }}$ correspond to eigenenergies

$$
E_{S_{T}}=\frac{J}{2}\left[S_{T}\left(S_{T}+1\right)-\frac{3 N}{4}\right]
$$


where $S_{T}$ is the eigenvalue of the total spin. From Eqs. (22), for a homogenized $S_{T}=0$ state, we get

$$
E_{S_{T}}=J \sum_{i, j>i}\left\langle\vec{S}_{i} \cdot \vec{S}_{j}\right\rangle=\frac{3 J N(N-1)}{2}\left\langle S_{i}^{z} S_{j}^{z}\right\rangle,
$$

which establishes the connection between $E_{S_{T}}$ and Eq. (9) through Eq. (5). Now, any VB state is an eigenstate of $H_{\text {IRHM }}$ [4]. Since, Rumer states are also VB states, a homogenized linear combination of non-crossing Rumer states is also an eigenstate of IRHM with entanglement given by Eq. (9).

Here, we should mention that the well-known Lipkin-Meshkov-Glick model (for a certain choice of parameters) [27] is a special case of the IRHM. The IRHM with two spins and four spins (with spins at the corners of a regular tetrahedron) can be realized from a Hubbard model. It has been shown that a zigzag graphene nanodisc can be described well by a long-range ferromagnetic Heisenberg model [28]. Moreover, the fully connected network (which can be mapped onto a spin system with distance-independent spin-spin interaction) is a well-studied model in the context of excitation energy transfer in light-harvesting complexes [29].

\section{Conclusions.}

In a VB state, both the spins of any two-spin singlet $(|\uparrow \downarrow\rangle-\mid \downarrow \uparrow$ ) ) are completely unentangled with the rest of the system. However, by using a homogenized superposition of VB states, we managed to distribute entanglement efficiently such that any pair is maximally entangled with the rest of the RVB system while concomitantly the constituent spins of the pair are completely unentangled with each other. Now, we know from Lieb-Mattis theorem [30] that states with total spin zero are quite commonly ground states of interacting spin systems. However, it has not been recognized that one can generate high bipartite $E_{v}^{2}$ entanglement from such states. Our RVB

states with maximal $E_{v}^{2}$ can be realized physically in systems such as the infinite-range, large $U / t$ Hubbard model and infinite-range, hard-core-boson model with frustrated hopping [31] when they are at half-filling.

\section{Acknowledgments}

One of the authors (S. Y.) would like to thank G. Baskaran, R. Simon, and S. Ghosh for valuable discussions. 


\section{References}

[1] C. K. Majumdar, J. Phys. C 3 (1969) 911; C. K. Majumdar, D. K. Ghosh, J. Math. Phys. 10 (1969) 1388.

[2] B. S. Shastry and B. Sutherland, Phys. Rev. Lett. 47 (1981) 964.

[3] L. Hulthén, Ark. Mat., Astron. Fys. 26 (1938) 1; M. Mambrini, Phys. Rev. B 77 (2008) 134430.

[4] See supplementary material for details.

[5] P. Fazekas, P. W. Anderson, Philos. Mag. 30 (1974) 423; G. Misguich, C. Lhuillier, in: H. T. Diep (Ed.), Frustrated Spin Systems, WorldScientific, 2003.

[6] P. W. Anderson, Science 235 (1987) 1196.

[7] P. W. Anderson, G. Baskaran, Z. Zou, T. Hsu, Phys. Rev. Lett. 58 (1987) 2790.

[8] P. A. Lee, N. Nagaosa, X.-G. Wen, Rev. Mod. Phys. 78 (2006) 17.

[9] T. Ishiguro, K. Yamaji, G. Saito, Organic Superconductors Springer, New York, 1998.

[10] E. A. Ekimov, V. A. Sidorov, E. D. Bauer, N. N. Mel'nik, N. J. Curro, J. D. Thompson, S. M. Stishov, Nature (London) 428 (2004) 542.

[11] A. Y. Kitaev, Ann. Phys. (N.Y.) 303 (2003) 2.

[12] D. Das, H. Singh, T. Chakraborty, R. K. Gopal and C. Mitra, New J. Phys. 15 (2013) 013047.

[13] S. Miyahara and K. Ueda, J. Phys.: Condens. Matter 15 (2003) R327.

[14] B.S. Shastry and B. Sutherland, Physica B 108 (1981) 1069.

[15] S. Sachdev, Nature Phys. 4 (2008) 173.

[16] J. I. Latorre, A. Riera, J. Phys. A: Math. Theor. 42 (2009) 504002.

[17] G. Vidal, Phys. Rev. Lett. 91 (2003) 147902. 
[18] S. Liang, P. W. Anderson, Phy. Rev. Lett. 61 (1998) 365.

[19] A. Chandran, D. Kaszilkowski, A. Sen(De), U. Sen, V. Vedral, Phys. Rev. Lett. 99 (2007) 170502; D. Kaszlikowski, Aditi Sen(De), Ujjwal Sen, V. Vedral, Phys. Rev. Lett. 101 (2008) 248902; F. Alet, D.Braun, G. Misguich, Phy. Rev. Lett. 101 (2008) 248901.

[20] U. Glaser, H. Büttner, H. Fehske, Phys. Rev. A. 68 (2003) 032318.

[21] V. Coffman, J. Kundu, W. K. Wootters, Phys. Rev. A 61 (2000) 052306;

T. J. Osborne, F. Verstraete, Phys. Rev. Lett. 96 (2006) 220503.

[22] A. Higuchi, A. Sudbery, Phys. Lett. A 273 (2000) 213.

[23] A. Borras, A. R. Plastino, J. Batle, C. Zander, M. Casas, A. Plastino, J. Phys. A: Math. Theor. 40 (2007) 13407.

[24] P. Rungta, V. Bužek, Carlton M. Caves, M. Hillery, G. J. Milburn, Phys. Rev. A 64 (2001) 042315.

[25] L. Pauling, J. Chem. Phys. 1 (1933) 280.

[26] G. Rumer, Nachr. d. Ges. d. Wiss. zu Gottingen, M. P. Klasse (1932) 337.

[27] H. J. Lipkin, N. Meshkov, and A. J. Glick, Nucl. Phys. 62 (1965) 188.

[28] M. Ezawa, New J. of Phys. 11 (2009) 095005.

[29] A W Chin, A Datta, F Caruso1, S F Huelga1, and M. B. Plenio, New J. Phys. 12 (2010) 065002.

[30] E. Lieb, D. Mattis, Phys. Rev. 125 (1962) 164.

[31] For examples of frustrated hopping, see F. Wang, F. Pollmann, A. Vishwanath, Phys. Rev. Lett. 102 (2009) 017203. 


\section{Supplemental Material for "Study of two-spin entanglement in sin- glet states"}

\section{Singlet as a superposition of valence-bond states}

We will sketch an argument showing that any spin-singlet state can be expressed as a superposition of valence-bond (VB) states.

We first note that there are $(N-1) ! !\left(=(N !) /\left[2^{N / 2}(N / 2) !\right]\right)$ VB states each of which is a product of $N / 2$ dimer states of the form $|\uparrow \downarrow\rangle-|\downarrow \uparrow\rangle$ (with no pair of dimers sharing a spin); thus, each VB state has total spin eigenvalue $S_{T}=0$. It is worth noting that

$$
\begin{aligned}
\left|\Phi_{13}^{S_{13}=0}\right\rangle \otimes\left|\Phi_{24}^{S_{24}=0}\right\rangle= & \left|\Phi_{12}^{S_{12}=0}\right\rangle \otimes\left|\Phi_{34}^{S_{34}=0}\right\rangle \\
& +\left|\Phi_{14}^{S_{14}=0}\right\rangle \otimes\left|\Phi_{23}^{S_{23}=0}\right\rangle
\end{aligned}
$$

where $\left|\Phi_{i j}^{S_{i j}=0}\right\rangle \equiv\left[|\uparrow\rangle_{i}|\downarrow\rangle_{j}-|\downarrow\rangle_{i}|\uparrow\rangle_{j}\right]$ is a dimer for spins at sites $i$ and $j$ with $S_{i j}$ being the total spin of $S_{i}$ and $S_{j}$. Using the above relation one can establish that there are only ${ }^{N} C_{\frac{N}{2}}-{ }^{N} C_{\frac{N}{2}-1}$ linearly-independent VB states 1]. A particular set of linearly-independent VB states are the Rumer states [1, 2] that are made up of non-crossing dimers.

Next, we note that a spin eigenfunction with total spin $S_{T}$ (for a spin system with $\mathrm{N}$ spins) is obtained from the (N-1) electron eigenfunctions by adding or subtracting the spin of the last electron. Then, the degeneracy $g\left(N, S_{T}\right)$ of the spin $S_{T}$ state in a N-spin system is given by

$$
g\left(N, S_{T}\right)=g\left(N-1, S_{T}-\frac{1}{2}\right)+g\left(N-1, S_{T}+\frac{1}{2}\right) .
$$

Then, by induction, it follows that [3]

$$
g\left(N, S_{T}\right)={ }^{N} C_{\frac{N}{2}-S_{T}}-{ }^{N} C_{\frac{N}{2}-S_{T}-1} .
$$

Thus, the number of linearly-independent spin-singlets (i.e., spin eigenfunctions with $\left.S_{T}=0\right)$ is ${ }^{N} C_{\frac{N}{2}}-{ }^{N} C_{\frac{N}{2}-1}$ which is the same as the number of linearly-independent VB states. Thus, any singlet can be expressed as a linear superposition of VB states.

Of interest are various types of VB coverings of a lattice. The equalamplitude superposition of all nearest-neighbor-singlet VB coverings is a disordered RVB liquid state. When time-reversal symmetry is broken in 
a valence-bond spin liquid a chiral spin liquid is realized. On the other hand, when lattice symmetry is spontaneously broken, bond-ordered VB solids result.

\section{Construction of maximal $E_{v}^{2}$ states for six spins}

As mentioned in the main text, we show two different ways to construct maximal $E_{v}^{2}$ states.

\subsection{Generating homogeneity in six-spin, isotropic states}

There are five linearly-independent states for the case of six spins; they correspond to the five non-crossing Rumer diagrams of Fig. 3 in the main text. Similar to the four-spin case, we begin with the following general linear superposition (mentioned in the main text):

$$
\begin{aligned}
\left|\Psi^{6}\right\rangle & =r_{1} e^{i \theta_{1}}\left[\left|\Phi_{12}^{S_{12}=0}\right\rangle \otimes\left|\Phi_{34}^{S_{34}=0}\right\rangle \otimes\left|\Phi_{56}^{S_{56}=0}\right\rangle\right] \\
& +r_{2} e^{i \theta_{2}}\left[\left|\Phi_{61}^{S_{61}=0}\right\rangle \otimes\left|\Phi_{23}^{S_{23}=0}\right\rangle \otimes\left|\Phi_{45}^{S_{45}=0}\right\rangle\right] \\
& +r_{3} e^{i \theta_{3}}\left[\left|\Phi_{12}^{S_{12}=0}\right\rangle \otimes\left|\Phi_{36}^{S_{36}=0}\right\rangle \otimes\left|\Phi_{45}^{S_{45}=0}\right\rangle\right] \\
& +r_{4} e^{i \theta_{4}}\left[\left|\Phi_{23}^{S_{23}=0}\right\rangle \otimes\left|\Phi_{14}^{S_{14}=0}\right\rangle \otimes\left|\Phi_{56}^{S_{56}=0}\right\rangle\right] \\
& +r_{5} e^{i \theta_{5}}\left[\left|\Phi_{16}^{S_{16}=0}\right\rangle \otimes\left|\Phi_{25}^{S_{25}=0}\right\rangle \otimes\left|\Phi_{34}^{S_{34}=0}\right\rangle\right],
\end{aligned}
$$

and obtain solutions that homogenize $\left|\Psi^{6}\right\rangle$, i.e., choose appropriate coefficients in $\left|\Psi^{6}\right\rangle$ so as to get the form $\left|\Psi_{6}\right\rangle_{\text {hom }}$ mentioned in Eq. (11) of the main text. Next, we evaluate Eq. (S4) and get the coefficients for the various basis states $\left|\psi_{k}^{z}\right\rangle$ in Eq. (11) of the main text. The expressions for the coefficients $e^{i \delta_{k}}$ in Eq. (11) of the main text are shown in Table S1. Similar to the four-spin case, we make the state in Eq. (S4) homogeneous.

Since there are only five linearly-independent Rumer states, we can construct only five linearly-independent superpositions of the form of $\left|\Psi^{6}\right\rangle$ given in Eq. (S4). Thus, we can expect that five independent sets of coefficients $r_{i} e^{i \theta_{i}}$ will produce homogeneity. Here, we present five such linearlyindependent solutions:

$$
\begin{aligned}
& \text { 1. }\left\{r_{1}=r_{2}=0 ;\left(r_{3} e^{i \theta_{3}}, r_{4} e^{i \theta_{4}}, r_{5} e^{i \theta_{5}}\right)=\left(\omega_{4}, \omega_{4}^{2}, \omega_{4}^{3}\right)\right\}, \\
& \text { 2. }\left\{r_{1}=r_{2}=0 ;\left(r_{3} e^{i \theta_{3}}, r_{4} e^{i \theta_{4}}, r_{5} e^{i \theta_{5}}\right)=\left(\omega_{4}^{*}, \omega_{4}^{* 2}, \omega_{4}^{* 3}\right)\right\},
\end{aligned}
$$




\begin{tabular}{|c|c|c|c|c|c|}
\hline Basis states $\psi_{k}^{z}$ & \multicolumn{5}{|c|}{ Coefficients $e^{i \delta_{k}}$ in Eq. (11) of main text. } \\
\hline$|\uparrow \downarrow \uparrow \downarrow \uparrow \downarrow\rangle$ & $r_{1} e^{i \theta_{1}}$ & $-r_{2} e^{i \theta_{2}}$ & $-r_{3} e^{i \theta_{3}}$ & $-r_{4} e^{i \theta_{4}}$ & $-r_{5} e^{i \theta_{5}}$ \\
\hline$|\downarrow \uparrow \uparrow \downarrow \uparrow \downarrow\rangle$ & $-r_{1} e^{i \theta_{1}}$ & & $+r_{3} e^{i \theta_{3}}$ & & \\
\hline$|\uparrow \downarrow \downarrow \uparrow \uparrow \downarrow\rangle$ & $-r_{1} e^{i \theta_{1}}$ & & & & $+r_{5} e^{i \theta_{5}}$ \\
\hline$|\downarrow \uparrow \downarrow \uparrow \uparrow \downarrow\rangle$ & $r_{1} e^{i \theta_{1}}$ & & & $-r_{4} e^{i \theta_{4}}$ & \\
\hline$|\uparrow \downarrow \uparrow \downarrow \downarrow \uparrow\rangle$ & $-r_{1} e^{i \theta_{1}}$ & & & $+r_{4} e^{i \theta_{4}}$ & \\
\hline$|\downarrow \uparrow \uparrow \downarrow \downarrow \uparrow\rangle$ & $r_{1} e^{i \theta_{1}}$ & & & & $-r_{5} e^{i \theta_{5}}$ \\
\hline$|\uparrow \downarrow \downarrow \uparrow \downarrow \uparrow\rangle$ & $r_{1} e^{i \theta_{1}}$ & & $-r_{3} e^{i \theta_{3}}$ & & \\
\hline$|\downarrow \uparrow \downarrow \uparrow \downarrow \uparrow\rangle$ & $-r_{1} e^{i \theta_{1}}$ & $+r_{2} e^{i \theta_{2}}$ & $+r_{3} e^{i \theta_{3}}$ & $+r_{4} e^{i \theta_{4}}$ & $+r_{5} e^{i \theta_{5}}$ \\
\hline$|\uparrow \uparrow \downarrow \downarrow \uparrow \downarrow\rangle$ & & $+r_{2} e^{i \theta_{2}}$ & & $+r_{4} e^{i \theta_{4}}$ & \\
\hline$|\uparrow \uparrow \downarrow \downarrow \downarrow \uparrow\rangle$ & & & & $-r_{4} e^{i \theta_{4}}$ & \\
\hline$|\downarrow \downarrow \uparrow \uparrow \uparrow \downarrow\rangle$ & & & & $+r_{4} e^{i \theta_{4}}$ & \\
\hline$|\downarrow \downarrow \uparrow \uparrow \downarrow \uparrow\rangle$ & & $-r_{2} e^{i \theta_{2}}$ & & $-r_{4} e^{i \theta_{4}}$ & \\
\hline$|\downarrow \downarrow \downarrow \uparrow \uparrow \uparrow\rangle$ & & & & & $-r_{5} e^{i \theta_{5}}$ \\
\hline$|\uparrow \uparrow \uparrow \downarrow \downarrow \downarrow\rangle$ & & & & & $+r_{5} e^{i \theta_{5}}$ \\
\hline$|\uparrow \uparrow \downarrow \uparrow \downarrow \downarrow\rangle$ & & $-r_{2} e^{i \theta_{2}}$ & & & $-r_{5} e^{i \theta_{5}}$ \\
\hline$|\downarrow \downarrow \uparrow \downarrow \uparrow \uparrow\rangle$ & & $+r_{2} e^{i \theta_{2}}$ & & & $+r_{5} e^{i \theta_{5}}$ \\
\hline$|\downarrow \uparrow \uparrow \uparrow \downarrow \downarrow\rangle$ & & & $-r_{3} e^{i \theta_{3}}$ & & \\
\hline$|\uparrow \downarrow \downarrow \downarrow \uparrow \uparrow\rangle$ & & & $+r_{3} e^{i \theta_{3}}$ & & \\
\hline$|\downarrow \uparrow \downarrow \downarrow \uparrow \uparrow\rangle$ & & $-r_{2} e^{i \theta_{2}}$ & $-r_{3} e^{i \theta_{3}}$ & & \\
\hline$|\uparrow \downarrow \uparrow \uparrow \downarrow \downarrow\rangle$ & & $+r_{2} e^{i \theta_{2}}$ & $+r_{3} e^{i \theta_{3}}$ & & \\
\hline
\end{tabular}

Table S1: Basis states $\left|\psi_{k}^{z}\right\rangle$ of Eq. (11) in the main text for a six-spin system and the corresponding coefficients $e^{i \delta_{k}}$ determined from the equation $\left|\Psi^{6}\right\rangle=\left|\Psi_{6}\right\rangle_{\text {hom }}$. 
3. $\left\{r_{1}=r_{2}=0 ;\left(r_{3} e^{i \theta_{3}}, r_{4} e^{i \theta_{4}}, r_{5} e^{i \theta_{5}}\right)=(-1,+1,-1)\right\}$,

4. $\left\{r_{1} e^{i \theta_{1}}=-1+e^{i \alpha} ; r_{2} e^{i \theta_{2}}=1+e^{i \alpha}\right.$;

$$
\left.\left(r_{3} e^{i \theta_{3}}, r_{4} e^{i \theta_{4}}, r_{5} e^{i \theta_{5}}\right)=(-1,-1,-1)\right\}
$$

5. $\left\{r_{1} e^{i \theta_{1}}=-1+e^{-i \alpha} ; r_{2} e^{i \theta_{2}}=1+e^{-i \alpha}\right.$;

$$
\left.\left(r_{3} e^{i \theta_{3}}, r_{4} e^{i \theta_{4}}, r_{5} e^{i \theta_{5}}\right)=(-1,-1,-1)\right\},
$$

where $\omega_{4}=e^{i 2 \pi / 4}$ is a fourth root of unity and $e^{i \alpha}$ is a complex number. In arriving at the above solutions, we used the fact that when sum of four unit vectors is a zero vector (i.e., when $\sum_{j=1}^{4} e^{i \alpha_{j}}=0$ ), one pair of the unit vectors will add up to a zero vector with the remaining pair also producing a zero vector. In Fig. S1, we show one of the three possibilities for $\sum_{j=1}^{4} e^{i \alpha_{j}}=0$. Out of the five solutions given above, the first and the second solutions produce the states (as mentioned in the main text)

$$
\begin{aligned}
\left|\Psi_{a}^{6}\right\rangle= & \omega_{4}\left(\left|\Phi_{12}^{S_{12}=0}\right\rangle \otimes\left|\Phi_{36}^{S_{36}=0}\right\rangle \otimes\left|\Phi_{45}^{S_{45}=0}\right\rangle\right) \\
& +\omega_{4}^{2}\left(\left|\Phi_{23}^{S_{23}=0}\right\rangle \otimes\left|\Phi_{14}^{S_{14}=0}\right\rangle \otimes\left|\Phi_{56}^{S_{56}=0}\right\rangle\right) \\
& +\omega_{4}^{3}\left(\left|\Phi_{16}^{S_{16}=0}\right\rangle \otimes\left|\Phi_{25}^{S_{25}=0}\right\rangle \otimes\left|\Phi_{34}^{S_{34}=0}\right\rangle\right),
\end{aligned}
$$

and its complex conjugate, respectively, whereas the third solution yields the state (as given in the main text)

$$
\begin{aligned}
\left|\Psi_{b}^{6}\right\rangle= & -\left(\left|\Phi_{12}^{S_{12}=0}\right\rangle \otimes\left|\Phi_{36}^{S_{36}=0}\right\rangle \otimes\left|\Phi_{45}^{S_{45}=0}\right\rangle\right) \\
& +\left(\left|\Phi_{23}^{S_{23}=0}\right\rangle \otimes\left|\Phi_{14}^{S_{14}=0}\right\rangle \otimes\left|\Phi_{56}^{S_{56}=0}\right\rangle\right) \\
& -\left(\left|\Phi_{16}^{S_{16}=0}\right\rangle \otimes\left|\Phi_{25}^{S_{25}=0}\right\rangle \otimes\left|\Phi_{34}^{S_{34}=0}\right\rangle\right) .
\end{aligned}
$$

The states that are obtained from the fourth and the fifth solutions can be written as

$$
\begin{aligned}
\left|\Psi_{c}^{6}\right\rangle= & \left(-1+e^{i \alpha}\right)\left[\left|\Phi_{12}^{S_{12}=0}\right\rangle \otimes\left|\Phi_{34}^{S_{34}=0}\right\rangle \otimes\left|\Phi_{56}^{S_{56}=0}\right\rangle\right] \\
& +\left(1+e^{i \alpha}\right)\left[\left|\Phi_{61}^{S_{61}=0}\right\rangle \otimes\left|\Phi_{23}^{S_{23}=0}\right\rangle \otimes\left|\Phi_{45}^{S_{45}=0}\right\rangle\right] \\
& -\left[\left|\Phi_{12}^{S_{12}=0}\right\rangle \otimes\left|\Phi_{36}^{S_{36}=0}\right\rangle \otimes\left|\Phi_{45}^{S_{45}=0}\right\rangle\right] \\
& -\left[\left|\Phi_{23}^{S_{23}=0}\right\rangle \otimes\left|\Phi_{14}^{S_{14}=0}\right\rangle \otimes\left|\Phi_{56}^{S_{56}=0}\right\rangle\right] \\
& -\left[\left|\Phi_{16}^{S_{16}=0}\right\rangle \otimes\left|\Phi_{25}^{S_{25}=0}\right\rangle \otimes\left|\Phi_{34}^{S_{34}=0}\right\rangle\right]
\end{aligned}
$$


and its complex conjugate, respectively. Using $e^{i \alpha}=-i\left(=-\omega_{4}\right)$, the state in Eq. (S7) can be rewritten as

$$
\begin{aligned}
\left|\Psi_{c}^{6}\right\rangle= & \omega_{4}\left(\left|\Phi_{12}^{S_{12}=0}\right\rangle \otimes\left|\Phi_{34}^{S_{34}=0}\right\rangle \otimes\left|\Phi_{65}^{S_{65}=0}\right\rangle\right) \\
& +\omega_{4}^{2}\left(\left|\Phi_{14}^{S_{14}=0}\right\rangle \otimes\left|\Phi_{25}^{S_{25}=0}\right\rangle \otimes\left|\Phi_{36}^{S_{36}=0}\right\rangle\right) \\
& +\omega_{4}^{3}\left(\left|\Phi_{61}^{S_{61}=0}\right\rangle \otimes\left|\Phi_{23}^{S_{23}=0}\right\rangle \otimes\left|\Phi_{45}^{S_{45}=0}\right\rangle\right) .
\end{aligned}
$$

The above state represents the superposition of the three diagrams of Fig. $3(\mathrm{a})$ in the main text.

Evaluating the tensor products in the above Eqs. (S5), (S6), and (S8), we rewrite these states as

$$
\begin{aligned}
&\left|\Psi_{a}^{6}\right\rangle=(|\uparrow \downarrow \uparrow \downarrow \uparrow \downarrow\rangle-|\downarrow \uparrow \downarrow \uparrow \downarrow \uparrow\rangle) \\
&+ \omega_{4}(|\uparrow \downarrow \uparrow \uparrow \downarrow \downarrow\rangle+|\uparrow \downarrow \downarrow \downarrow \uparrow \uparrow\rangle+|\downarrow \uparrow \uparrow \downarrow \uparrow \downarrow\rangle \\
&-|\uparrow \downarrow \downarrow \uparrow \downarrow \uparrow\rangle-|\downarrow \uparrow \uparrow \uparrow \downarrow \downarrow\rangle-|\downarrow \uparrow \downarrow \downarrow \uparrow \uparrow\rangle) \\
&+\omega_{4}^{2}(|\uparrow \uparrow \downarrow \downarrow \uparrow \downarrow\rangle+|\uparrow \downarrow \uparrow \downarrow \downarrow \uparrow\rangle+|\downarrow \downarrow \uparrow \uparrow \uparrow \downarrow\rangle \\
&-|\uparrow \uparrow \downarrow \downarrow \downarrow \uparrow\rangle-|\downarrow \uparrow \downarrow \uparrow \uparrow \downarrow\rangle-|\downarrow \downarrow \uparrow \uparrow \downarrow \uparrow\rangle) \\
&+\omega_{4}^{3}(|\uparrow \uparrow \uparrow \downarrow \downarrow \downarrow\rangle+|\downarrow \downarrow \uparrow \downarrow \uparrow \uparrow\rangle+|\uparrow \downarrow \downarrow \uparrow \uparrow \downarrow\rangle \\
&-|\uparrow \uparrow \downarrow \uparrow \downarrow \downarrow\rangle-|\downarrow \uparrow \uparrow \downarrow \downarrow \uparrow\rangle-|\downarrow \downarrow \downarrow \uparrow \uparrow \uparrow\rangle)], \\
& \quad-(|\uparrow \downarrow \uparrow \uparrow \downarrow \downarrow\rangle+|\uparrow \downarrow \downarrow \downarrow \uparrow \uparrow+| \downarrow \uparrow \uparrow \downarrow \uparrow \downarrow\rangle \\
&-|\uparrow \downarrow \downarrow \uparrow \downarrow \uparrow\rangle-|\downarrow \uparrow \uparrow \uparrow \downarrow \downarrow\rangle-|\downarrow \uparrow \downarrow \downarrow \uparrow \uparrow\rangle) \\
&+(|\uparrow \uparrow \downarrow \downarrow \uparrow \downarrow\rangle+|\uparrow \downarrow \uparrow \downarrow \downarrow \uparrow\rangle+|\downarrow \downarrow \uparrow \uparrow \uparrow \downarrow\rangle \\
&-\left|\Psi_{b}^{6}\right\rangle=\begin{aligned}
(|\uparrow \downarrow \uparrow \downarrow \uparrow \downarrow\rangle-|\downarrow \uparrow \downarrow \uparrow \downarrow \uparrow\rangle) \\
-(|\uparrow \uparrow \uparrow \downarrow \downarrow \downarrow\rangle+|\downarrow \downarrow \uparrow \downarrow \uparrow \uparrow\rangle+|\uparrow \downarrow \downarrow \uparrow \uparrow \downarrow\rangle
\end{aligned} \\
&-|\uparrow \uparrow \downarrow \uparrow \downarrow \downarrow\rangle-|\downarrow \uparrow \uparrow \downarrow \downarrow \uparrow\rangle-|\downarrow \downarrow \downarrow \uparrow \uparrow \uparrow\rangle)],
\end{aligned}
$$

and

$$
\begin{aligned}
\left|\Psi_{c}^{6}\right\rangle= & {[(|\uparrow \downarrow \uparrow \downarrow \uparrow \downarrow\rangle-|\downarrow \uparrow \downarrow \uparrow \downarrow \uparrow\rangle)} \\
& +\omega_{4}(|\uparrow \downarrow \uparrow \downarrow \downarrow \uparrow\rangle+|\uparrow \downarrow \downarrow \uparrow \uparrow \downarrow\rangle+|\downarrow \uparrow \uparrow \downarrow \uparrow \downarrow\rangle
\end{aligned}
$$




$$
\begin{gathered}
-|\uparrow \downarrow \downarrow \uparrow \downarrow \uparrow\rangle-|\downarrow \uparrow \uparrow \downarrow \downarrow \uparrow\rangle-|\downarrow \uparrow \downarrow \uparrow \uparrow \downarrow\rangle) \\
+\omega_{4}^{2}(|\uparrow \uparrow \uparrow \downarrow \downarrow \downarrow\rangle+|\uparrow \downarrow \downarrow \downarrow \uparrow \uparrow\rangle+|\downarrow \downarrow \uparrow \uparrow \uparrow \downarrow\rangle \\
-|\uparrow \uparrow \downarrow \downarrow \downarrow \uparrow\rangle-|\downarrow \uparrow \uparrow \uparrow \downarrow \downarrow\rangle-|\downarrow \downarrow \downarrow \uparrow \uparrow \uparrow\rangle) \\
+\omega_{4}^{3}(|\uparrow \uparrow \downarrow \downarrow \uparrow \downarrow\rangle+|\uparrow \downarrow \uparrow \uparrow \downarrow \downarrow\rangle+|\downarrow \downarrow \uparrow \downarrow \uparrow \uparrow\rangle \\
-|\uparrow \uparrow \downarrow \uparrow \downarrow \downarrow\rangle-|\downarrow \uparrow \downarrow \downarrow \uparrow \uparrow\rangle-|\downarrow \downarrow \uparrow \uparrow \downarrow \uparrow\rangle)] .
\end{gathered}
$$

\subsection{Producing isotropy in six-spin, homogeneous state}

A homogeneous state with six spins can be written as follows:

$$
\begin{aligned}
\left|\Psi_{6}\right\rangle_{h o m}= & e^{i \phi_{1}}[|\uparrow \downarrow \uparrow \downarrow \uparrow \downarrow\rangle-|\downarrow \uparrow \downarrow \uparrow \downarrow \uparrow\rangle] \\
& +e^{i \phi_{2}}[|\downarrow \uparrow \uparrow \downarrow \uparrow \downarrow\rangle-|\uparrow \downarrow \downarrow \uparrow \downarrow \uparrow\rangle] \\
& +e^{i \phi_{3}}[|\uparrow \downarrow \uparrow \uparrow \uparrow\rangle-|\downarrow \uparrow \uparrow \downarrow \downarrow \uparrow\rangle] \\
& +e^{i \phi_{4}}[|\downarrow \uparrow \downarrow \uparrow \uparrow \downarrow\rangle-|\uparrow \downarrow \uparrow \downarrow \downarrow \uparrow\rangle] \\
& \left.+e^{i \phi_{5}}[|\uparrow \uparrow \downarrow \uparrow \uparrow \downarrow-| \downarrow \downarrow \uparrow \uparrow \downarrow \uparrow\rangle\right] \\
& +e^{i \phi_{6}}[|\uparrow \uparrow \downarrow \downarrow \uparrow\rangle-|\downarrow \downarrow \uparrow \uparrow \uparrow \downarrow\rangle] \\
& +e^{i \phi_{7}}[|\downarrow \downarrow \uparrow \uparrow \uparrow\rangle-|\uparrow \uparrow \uparrow \downarrow \downarrow \downarrow\rangle] \\
& +e^{i \phi_{8}}[|\uparrow \uparrow \downarrow \uparrow \downarrow \downarrow\rangle-|\downarrow \downarrow \uparrow \downarrow \uparrow \uparrow\rangle] \\
& +e^{i \phi_{9}}[|\downarrow \uparrow \uparrow \uparrow \downarrow \downarrow\rangle-|\uparrow \downarrow \downarrow \downarrow \uparrow \uparrow\rangle] \\
& +e^{i \phi_{10}}[|\downarrow \uparrow \downarrow \downarrow \uparrow \uparrow\rangle-|\uparrow \downarrow \uparrow \uparrow \downarrow \downarrow\rangle] .
\end{aligned}
$$

For the above homogeneous state, the isotropy condition (expressed as $\sum_{i=1}^{6} S_{i}^{+}\left|\Psi_{N}\right\rangle_{h o m}=$ 0 ) yields the various basis states and the corresponding coefficients shown in Table \$2. The set of fifteen equations (corresponding to the zero-valued coefficients of the basis states) in Table $\$ 2$ can be reduced to the following set of five linearly-independent equations:

$$
\begin{aligned}
& e^{i \phi_{1}}+e^{i \phi_{2}}+e^{i \phi_{5}}-e^{i \phi_{7}}=0, \\
& e^{i \phi_{1}}+e^{i \phi_{3}}-e^{i \phi_{6}}-e^{i \phi_{10}}=0, \\
& e^{i \phi_{1}}-e^{i \phi_{4}}-e^{i \phi_{8}}-e^{i \phi_{9}}=0, \\
& e^{i \phi_{1}}-e^{i \phi_{4}}-e^{i \phi_{7}}-e^{i \phi_{10}}=0, \\
& e^{i \phi_{1}}+e^{i \phi_{2}}-e^{i \phi_{6}}-e^{i \phi_{8}}=0 .
\end{aligned}
$$

Each of the above equations (S13)-(S17) can be viewed as the sum of four unit vectors on a circle in the complex plane. Since the sum is of the form $\sum_{j=1}^{4} e^{i \alpha_{j}}=0$, it implies the conditions shown in Fig. S1. A set of five 


\begin{tabular}{|l|l|}
\hline $\begin{array}{l}\text { Basis states } \\
\text { contained in }\end{array}$ & $\begin{array}{l}\text { Zero-valued coeffi- } \\
\text { cients of basis states } \\
\sum_{i=1}^{6} S_{i}^{+}\left|\Psi^{6}\right\rangle_{\text {hom }} . \\
\text { obtained from using } \\
\text { isotropy on }\left|\Psi_{6}\right\rangle_{\text {hom }}\end{array}$ \\
\hline$|\uparrow \uparrow \uparrow \downarrow \uparrow \downarrow\rangle$ & $e^{i \phi_{1}}+e^{i \phi_{2}}+e^{i \phi_{5}}-e^{i \phi_{7}}$ \\
\hline$|\uparrow \downarrow \uparrow \uparrow \uparrow \downarrow\rangle$ & $e^{i \phi_{1}}+e^{i \phi_{3}}-e^{i \phi_{6}}-e^{i \phi_{10}}$ \\
\hline$|\uparrow \downarrow \uparrow \downarrow \uparrow \uparrow\rangle$ & $e^{i \phi_{1}}-e^{i \phi_{4}}-e^{i \phi_{8}}-e^{i \phi_{9}}$ \\
\hline$|\downarrow \uparrow \uparrow \uparrow \uparrow \downarrow\rangle$ & $e^{i \phi_{2}}+e^{i \phi_{4}}+e^{i \phi_{9}}-e^{i \phi_{6}}$ \\
\hline$|\downarrow \uparrow \uparrow \downarrow \uparrow \uparrow\rangle$ & $e^{i \phi_{2}}-e^{i \phi_{3}}-e^{i \phi_{8}}+e^{i \phi_{10}}$ \\
\hline$|\uparrow \uparrow \downarrow \uparrow \uparrow \downarrow\rangle$ & $e^{i \phi_{3}}+e^{i \phi_{4}}+e^{i \phi_{5}}+e^{i \phi_{8}}$ \\
\hline$|\downarrow \uparrow \downarrow \uparrow \uparrow \uparrow\rangle$ & $-e^{i \phi_{1}}+e^{i \phi_{4}}+e^{i \phi_{7}}+e^{i \phi_{10}}$ \\
\hline$|\uparrow \uparrow \downarrow \downarrow \uparrow \uparrow\rangle$ & $e^{i \phi_{5}}+e^{i \phi_{6}}-e^{i \phi_{9}}+e^{i \phi_{10}}$ \\
\hline$|\uparrow \uparrow \uparrow \downarrow \downarrow \uparrow\rangle$ & $-e^{i \phi_{3}}-e^{i \phi_{4}}+e^{i \phi_{6}}-e^{i \phi_{7}}$ \\
\hline$|\uparrow \uparrow \downarrow \uparrow \downarrow \uparrow\rangle$ & $-e^{i \phi_{1}}-e^{i \phi_{2}}+e^{i \phi_{6}}+e^{i \phi_{8}}$ \\
\hline$|\uparrow \downarrow \downarrow \uparrow \uparrow \uparrow\rangle$ & $-e^{i \phi_{2}}+e^{i \phi_{3}}+e^{i \phi_{7}}-e^{i \phi_{9}}$ \\
\hline$|\downarrow \downarrow \uparrow \uparrow \uparrow \uparrow\rangle$ & $-e^{i \phi_{5}}-e^{i \phi_{6}}+e^{i \phi_{7}}-e^{i \phi_{8}}$ \\
\hline$|\uparrow \uparrow \uparrow \uparrow \downarrow \downarrow\rangle$ & $-e^{i \phi_{7}}+e^{i \phi_{8}}+e^{i \phi_{9}}-e^{i \phi_{10}}$ \\
\hline$|\downarrow \uparrow \uparrow \uparrow \downarrow \uparrow\rangle$ & $-e^{i \phi_{1}}-e^{i \phi_{3}}-e^{i \phi_{5}}+e^{i \phi_{9}}$ \\
\hline$|\uparrow \downarrow \uparrow \uparrow \downarrow \uparrow\rangle$ & $-e^{i \phi_{2}}-e^{i \phi_{4}}-e^{i \phi_{5}}-e^{i \phi_{10}}$ \\
\hline
\end{tabular}

Table S2: The basis states and the corresponding zero-valued coefficients obtained from the condition of isotropy $\sum_{i=1}^{6} S_{i}^{+}\left|\Psi_{6}\right\rangle_{\text {hom }}=0$. 


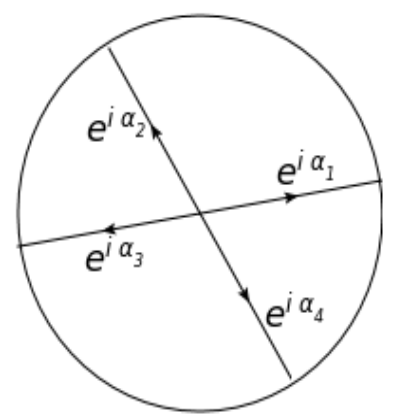

Figure S1: Geometric depiction of zero sum for four unit vectors on a unit circle in the complex plane. This represents one of the three possibilities of the zero $\operatorname{sum} \sum_{j=1}^{4} e^{i \alpha_{j}}=0$. The other two possibilities are $e^{i \alpha_{1}}+e^{i \alpha_{2}}=0, e^{i \alpha_{3}}+e^{i \alpha_{4}}=0$; and $e^{i \alpha_{1}}+e^{i \alpha_{4}}=0$, $e^{i \alpha_{2}}+e^{i \alpha_{3}}=0$.

\begin{tabular}{|c|c|c|c|c|c|}
\hline \multirow{2}{*}{$\begin{array}{l}\text { Coefficients in } \\
\text { Eqs. (S13)-(S17) }\end{array}$} & \multicolumn{5}{|c|}{ Five linearly independent solutions } \\
\hline & Sol. 1 & Sol. 2 & Sol. 3 & Sol. 4 & Sol. 5 \\
\hline$e^{i \phi_{1}}$ & 1 & 1 & 1 & 1 & 1 \\
\hline$e^{i \phi_{2}}$ & $\omega_{4}$ & $\omega_{4}^{*}$ & -1 & $-e^{i \alpha}$ & $-e^{-i \alpha}$ \\
\hline$e^{i \phi_{3}}$ & $\omega_{4}^{3}$ & $\omega_{4}^{* 3}$ & -1 & $-e^{i \alpha}$ & $-e^{-i \alpha}$ \\
\hline$e^{i \phi_{4}}$ & $-\omega_{4}^{2}$ & $-\omega_{4}^{* 2}$ & -1 & $e^{i \alpha}$ & $e^{-i \alpha}$ \\
\hline$e^{i \phi_{5}}$ & $\omega_{4}^{2}$ & $\omega_{4}^{* 2}$ & 1 & $e^{i \alpha}$ & $e^{-i \alpha}$ \\
\hline$e^{i \phi_{6}}$ & $-\omega_{4}^{2}$ & $-\omega_{4}^{* 2}$ & -1 & 1 & 1 \\
\hline$e^{i \phi_{7}}$ & $-\omega_{4}^{3}$ & $-\omega_{4}^{* 3}$ & 1 & 1 & 1 \\
\hline$e^{i \phi_{8}}$ & $-\omega_{4}^{3}$ & $-\omega_{4}^{* 3}$ & 1 & $-e^{i \alpha}$ & $-e^{-i \alpha}$ \\
\hline$e^{i \phi_{9}}$ & $-\omega_{4}$ & $-\omega_{4}^{*}$ & 1 & 1 & 1 \\
\hline$e^{i \phi_{10}}$ & $-\omega_{4}$ & $-\omega_{4}^{*}$ & 1 & $-e^{i \alpha}$ & $-e^{-i \alpha}$ \\
\hline
\end{tabular}

Table S3: Set of five linearly-independent solutions of Eqs. (S13)-(S17). 
linearly-independent solutions of Eqs. (S13)-(S17) is given in Table S3. This set of solutions produces the entangled states $\left|\Psi_{a}^{6}\right\rangle,\left|\Psi_{a}^{6 *}\right\rangle,\left|\Psi_{b}^{6}\right\rangle,\left|\Psi_{c}^{6}\right\rangle$, and $\left|\Psi_{c}^{6 *}\right\rangle$ which are the same as those obtained in the main text.

\section{Resonating-valence-bond picture}

Our maximal $E_{v}^{2}$ states, that form the ground states of IRHM, are a new class of RVB states made of homogenized superposition of VB states. We will now compare the entanglement properties of our RVB states and the general RVB states $|\Psi\rangle_{\mathrm{rvb}}$ of Ref. [4] given below:

$$
|\Psi\rangle_{\mathrm{rvb}}=\sum_{i_{\alpha} \in A ; j_{\beta} \in B} f\left(i_{1}, \ldots, i_{M}, j_{1}, \ldots, j_{M}\right)\left|\left(i_{1}, j_{1}\right) \ldots\left(i_{M}, j_{M}\right)\right\rangle
$$

where $M$ represents the number of sites in each sub-lattice and $f$ is assumed to be isotropic over the lattice. Also, $\left|\left(i_{k}, j_{k}\right)\right\rangle \equiv \frac{1}{\sqrt{2}}\left(|\uparrow\rangle_{i_{k}}|\downarrow\rangle_{j_{k}}-|\downarrow\rangle_{i_{k}}|\uparrow\rangle_{j_{k}}\right)$ denotes the singlet dimer connecting a site in sub-lattice $A$ with another site in sub-lattice $B$. The valence-bond basis states (used for the above RVB state $\left.|\Psi\rangle_{\mathrm{rvb}}\right)$ form an overcomplete set, whereas our RVB states are constructed from a complete set of ${ }^{N} C_{\frac{N}{2}}-{ }^{N} C_{\frac{N}{2}-1}$ states.

The rotational invariance of the two-spin reduced density matrix of a RVB state allows us to write it in the form of a Werner state [4]:

$$
\rho_{w}(p)=p|(i j)\rangle\langle(i j)|+\frac{1-p}{4} I_{4},
$$

where, for $1 / 3<p \leq 1$, the Werner state has the spins at site $i$ and site $j$ entangled with each other. In Ref. [4], an interesting analysis was carried out for the examples of RVB gas and RVB liquid. For the case of the RVB gas, the $|\Psi\rangle_{\mathrm{rvb}}$ state in Eq. (타) has $f$ as a constant (corresponding to equal-amplitude superposition of all bipartite valence-bond coverings). Consequently, based on the values of $p$, it was concluded that finite-size systems have a non-zero tangle (or entanglement) between the two sites [4]. Next, for the RVB liquid case involving equal-amplitude superposition of all nearestneighbor-singlet valence-bond coverings of a lattice, Monte Carlo calculations for a $4 \times 4$ lattice reveals zero (non-zero) tangle between the two sites for open (periodic) boundary conditions [4]. In contrast to this, our RVB states (with maximal $E_{v}^{2}$ ) yield zero entanglement between the two spins for all system sizes (as shown in Sec. II of the main text and below). It has been 
demonstrated that the $\mathrm{SU}(2)$ symmetry of the RVB states ensures that the two-spin correlation function and the parameter $p$ of the Werner state are related as [4]

$$
\left\langle\Psi\left|\vec{S}_{i} \cdot \vec{S}_{j}\right| \Psi\right\rangle=-\frac{3}{4} p
$$

Then, since our RVB states produce

$$
\left\langle S_{i}^{x} S_{j}^{x}\right\rangle=\left\langle S_{i}^{y} S_{j}^{y}\right\rangle=\left\langle S_{i}^{z} S_{j}^{z}\right\rangle=\frac{-1}{4(N-1)},
$$

it follows that $p=\frac{1}{N-1}$; thus, for systems with even number of spins $(N)$, we get zero entanglement between the two sites when $N \geq 4$. Therefore, we see that our RVB states (among the various RVB states), while producing maximum entanglement between a pair and the rest of the system, yield zero entanglement between the spins of that pair.

\section{Eigenstates of IRHM}

Here, we will show that any VB is an eigenstate of the IRHM Hamiltonian (mentioned in the main text)

$$
H_{\mathrm{IRHM}}=J \sum_{i, j>i} \vec{S}_{i} \cdot \vec{S}_{j}=\frac{J}{2}\left[\left(\sum_{i} \vec{S}_{i}\right)^{2}-\left(\sum_{i}{\overrightarrow{S_{i}}}^{2}\right)\right] .
$$

The eigenenergies of $H_{\text {IRHM }}$ are given by

$$
E_{S_{T}}=\frac{J}{2}\left[S_{T}\left(S_{T}+1\right)-\frac{3 N}{4}\right],
$$

where $S_{T}$ is the total spin eigenvalue. The ground state corresponds to $S_{T}=0$ which is rotationally invariant and also implies that $S_{T}^{z}=0$. Next, we note the interesting fact that

$$
\left[\overrightarrow{S_{1}} \cdot \overrightarrow{S_{3}}+\overrightarrow{S_{2}} \cdot \overrightarrow{S_{4}}+\overrightarrow{S_{1}} \cdot \overrightarrow{S_{4}}+\overrightarrow{S_{2}} \cdot \overrightarrow{S_{3}}\right]\left|\Phi_{12}^{S_{12}=0}\right\rangle \otimes\left|\Phi_{34}^{S_{34}=0}\right\rangle=0
$$

Since $\left|\Phi_{12}^{S_{12}=0}\right\rangle$ is an eigenstate of $\overrightarrow{S_{1}} \cdot \overrightarrow{S_{2}}$, it is obvious that $\left|\Phi_{12}^{S_{12}=0}\right\rangle \otimes\left|\Phi_{34}^{S_{34}=0}\right\rangle$ and the VB containing $\left|\Phi_{12}^{S_{12}=0}\right\rangle \otimes\left|\Phi_{34}^{S_{34}=0}\right\rangle$ are eigenstates of $\overrightarrow{S_{1}} \cdot \overrightarrow{S_{2}}+\overrightarrow{S_{3}} \cdot \overrightarrow{S_{4}}$. Hence, it is clear that $\left|\Phi_{12}^{S_{12}=0}\right\rangle \otimes\left|\Phi_{34}^{S_{34}=0}\right\rangle$ is an eigenstate of $\sum_{i=1,2,3,4 ; j>i} \vec{S}_{i} \cdot \vec{S}_{j}$. 
Then, for a system of $2 N$ spins, it follows by mathematical induction that a VB state involving $N$ dimers is an eigenstate of $H_{\text {IRHM }}$.

Alternately, from Eq. (S24), we note that a pair of dimers as well as a $\mathrm{VB}$ containing that pair of dimers are both eigenstates of the sum of the 4 inter-dimer interactions corresponding to the two dimers. For a system of $2 N$ spins, since there are ${ }^{N} C_{2}$ pairs of dimers in the $N$-dimer VB, there are $4 \times{ }^{N} C_{2}$ inter-dimer interactions in the $H_{\text {IRHM }}$; the $\mathrm{VB}$ is an eigenstate of the sum of the $4 \times{ }^{N} C_{2}$ inter-dimer interactions. Furthermore, the VB is also an eigenstate of the $N$ intra-dimer interactions. Now, there are only ${ }^{2 N} C_{2}$ spin interactions (in the $H_{\text {IRHM }}$ ) which is equal to the sum of $4 \times{ }^{N} C_{2}$ (number of inter-dimer interactions) and $N$ (number of intra-dimer interactions), i.e., ${ }^{2}{ }^{N} C_{2}=4 \times{ }^{N} C_{2}+N$. Thus, the $\mathrm{VB}$ is an eigenstate of $H_{\text {IRHM }}$.

\section{References}

[1] L. Pauling, J. Chem. Phys. 1 (1933) 280.

[2] G. Rumer, Nachr. d. Ges. d. Wiss. zu Gottingen, M. P. Klasse (1932) 337.

[3] R. Pauncz, The Construction of Spin Eigen-Functions: An Exercise Book (Kluwer Academic/Plenum Publishers, New York, 2000).

[4] A. Chandran, D. Kaszilkowski, A. Sen(De), U. Sen and V. Vedral, Phys. Rev. Lett. 99 (2007) 170502; D. Kaszlikowski, Aditi Sen(De), Ujjwal Sen, and V. Vedral, Phys. Rev. Lett. 101 (2008) 248902; F. Alet, D.Braun, and G. Misguich, Phy.Rev.Lett. 101 (2008) 248901. 